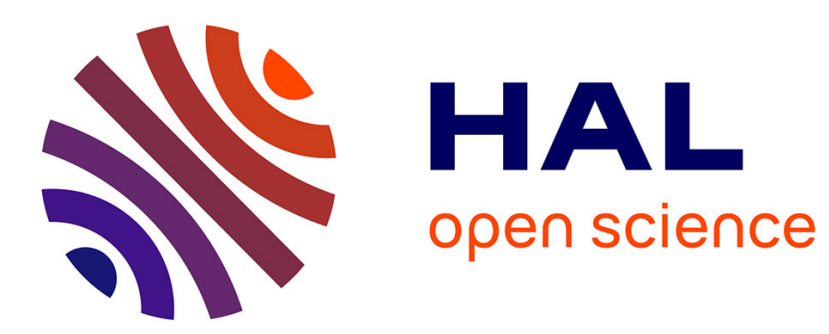

\title{
Coupling Continuous and Discontinuous Descriptions to Model First Body Deformation in Third Body Flows
}

Cao Hong-Phong, Mathieu Renouf, Frédéric Dubois, Yves Berthier

\section{To cite this version:}

Cao Hong-Phong, Mathieu Renouf, Frédéric Dubois, Yves Berthier. Coupling Continuous and Discontinuous Descriptions to Model First Body Deformation in Third Body Flows. Journal of Tribology, 2011, 133 (4), pp.041601-1 041601-7. 10.1115/1.4004881 . hal-00806618

\section{HAL Id: hal-00806618 https://hal.science/hal-00806618}

Submitted on 16 Jan 2017

HAL is a multi-disciplinary open access archive for the deposit and dissemination of scientific research documents, whether they are published or not. The documents may come from teaching and research institutions in France or abroad, or from public or private research centers.
L'archive ouverte pluridisciplinaire HAL, est destinée au dépôt et à la diffusion de documents scientifiques de niveau recherche, publiés ou non, émanant des établissements d'enseignement et de recherche français ou étrangers, des laboratoires publics ou privés. 


\title{
Coupling Continuous and Discontinuous Descriptions to Model First Body Deformation in Third Body Flows
}

\begin{abstract}
The present paper proposes an extension of the classical discrete element method used to study third body flows. Based on the concept of the tribological triplet proposed by Godet and Berthier, the aim of this work is to enrich description, by accounting for the deformation of the first body and investigating its influence on third-body rheology. To achieve this, a novel hybrid approach that combines continuous and discontinuous descriptions is used. To illustrate the advantage of such modeling, comparisons with the classical approach, which considers the first body as rigid, are performed in terms of mac-roscopic friction coefficient and velocity and stress profiles.
\end{abstract}

Keywords: friction, DEM-FEM coupling, NSCD, third body flows

Hong-Phong Cao

e-mail: hong-phong.cao@insa-lyon.fr

\author{
Mathieu Renouf \\ Associate Researcher \\ e-mail: Mathieu.Renouf@insa-lyon.fr
}

Laboratory of Mechanics of Contacts and Structures, University of Lyon, CNRS,

F-69621 Villeurbanne France

\author{
Frédéric Dubois \\ Research Engineer \\ Laboratory of Mechanics of Contacts and \\ Civil Engineering, \\ University of Montpellier II, CNRS, \\ F-34096 Montpellier France \\ e-mail: frederic.dubois@univ-montp2.fr
}

\author{
Yves Berthier \\ Research Director \\ Laboratory of Mechanics of Contacts and Structures, \\ University of Lyon, CNRS, \\ F-69621 Villeurbanne France \\ e-mail: Yves.Berthier@insa-lyon.fr
}

\section{Introduction}

Finding a solution to a tribological problem is a complex task. One of the main reasons for this complexity is the different scales involved in a contact, as they range from that of the mechanism ( $\mathrm{cm} \leq L \leq \mathrm{m})$ to that of the interface $(L \simeq \mu \mathrm{m})$, as underlined by Godet [1] and Berthier [2] in the concept of "tribological triplet." It is possible to study phenomena at the level of the mechanism [3], the bodies in contact [4], and the contact interface [5], but considering all these scales simultaneously remains difficult.

In the present paper, we focus on the contact interface, commonly called the third body, [6] in tribology. From a phenomenological viewpoint, the interface is a discontinuous layer that separates the bodies, known as first bodies, in contact. The third body is produced by the detachment of particles stemming from the first bodies and which mix with external particles introduced into the interface. While some of the roles of the third body have been clearly understood (velocity accommodation [5], charge loading capacity [6]), complete understanding of its rheology has not yet been achieved.

From the experimental viewpoint, it is difficult to observe a contact during a dynamical process without disturbing its behavior. Moreover, "post-mortem" analyses (for example, Scanning Electron Microscopy) do not give insight into what happens during the process, but merely give information on the final state of the bodies. Consequently, numerical tools have been developed to complete the information provided by experimental data.

To represent the evolution of the third body flows, two approaches are typically used. The first one is based on continuum theory $[7,8]$. Continuous approaches offer extensions of models derived from continuum mechanics and are used for modeling lubrication powders as well as granular lubrication, i.e., using an artificial third-body. They are used in many applications involving bearings [3], Couette geometries (annular) [9], parallel plates [10,11] and converging plates [7]. In the case of a natural third body (resulting for the most part from the bodies in contact), continuity hypotheses cannot be retained and, under such conditions, it is necessary to verify whether this approach is still valid and what its limits are. This has led to using the Discrete Element Method (DEM) to describe the discontinuity and heterogeneity of the third body, which is seen as a collection of cohesive particles interacting with their neighborhood. Elrod and Brewe [12] have underlined the capacity of such an approach to represent the evolution of the tribological interface. Several authors have investigated the influence of numerical parameters, geometry, and boundary conditions on the macroscopic behavior of the assembly $[13,14]$, and attention has been given to the influence of local cohesion and particle size on third body rheology and on macroscopic friction [15,16]. Fillot et al. [17] have extended such approaches to wear flows in simple shear simulation. More recently, Renouf et al. [18] used a cohesive zone model to create a bulk effect and describe wear flow in a fretting-like simulation. Kabir et al. [19] used deformable bodies instead of rigid bodies, but the system was too small and not dense enough to represent a real contact interface. All these works propose interesting phenomenological results, but an important point is still missing in the description of the interface, namely the deformation of the first bodies. This aspect must be taken into account in order to propose an accurate rheological model.

The present paper proposes a hybrid approach, combining both continuous description related to finite element modeling and discontinuous description related to rigid discrete element modeling to underline the influence of first body rigidity on third body rheology. This influence is illustrated in terms of velocity and stress profiles, global friction coefficient, and energy evolution.

\section{Mechanical Model}

The approach used in the present paper is based on the Non Smooth Contact Dynamics method (NSCD) developed by Moreau and Jean [20,21]. Based on a robust mathematical framework and using the same formalism, the approach is capable of 
representing the dynamical behavior of large collections of both rigid particles [22] and deformable particles [23]. Thus considering both rigid and deformable descriptions appears "natural" for this approach.

2.1 Mapping. Two linear mappings, $\mathbb{H}$ and its transpose $\mathbb{H}^{*}$, are used to transfer information from the contact frame (local level) to the body frame (global level) (cf. Fig. 1).

The local contact force vector $\mathbf{r}$ and the local relative velocity vector $\mathbf{u}$ are related to the global body contact force vector $\mathbf{R}$ and the body velocity vector $\dot{\mathbf{q}}$ by:

$$
\left\{\begin{array}{c}
\mathbf{R}=\mathbb{H} \mathbf{r} \\
\mathbf{u}=\mathbb{H}^{*} \dot{\mathbf{q}}
\end{array}\right.
$$

Both mappings depend on local information such as the local frame defined at each contact point and the network connectivity of each contact (cf. Fig. 1).

The hybrid contact element is the combination of a cell and a rigid half disk. When a particle is in contact with the half disk (cf. Fig. 1(a)), the contact force is divided into two parts and transferred to the two nodes A and B connected to the half disk. Thus the force on each node of the cell is computed by the following formula:

$$
\left\{\begin{array}{l}
F_{A}=F\left(\frac{d-d_{p}}{d}\right) \\
F_{B}=F\left(\frac{d_{p}}{d}\right)
\end{array}\right.
$$

where $d$ is the distance between $\mathrm{A}$ and $\mathrm{B}$ and $d_{p}$ is the distance between $\mathrm{A}$ and projection $\mathrm{P}$ of the contact point on segment $\mathrm{AB}$.

2.2 Equation of Motion. When the evolution of a multicontact system is smooth, the equation of dynamics can be written as:

$$
\mathbb{M} \ddot{\mathbf{q}}+\mathbf{F}^{i n t}(t, \mathbf{q}, \dot{\mathbf{q}})=\mathbf{F}^{e x t}(t, \mathbf{q}, \dot{\mathbf{q}})+\mathbf{R}
$$

where $\mathbb{M}$ represents the mass matrix, $\mathbf{F}^{\text {int }}(t, \mathbf{q}, \dot{\mathbf{q}})$ are the internal force and the nonlinear inertia terms, $\mathbf{F}^{\text {ext }}(t, \mathbf{q}, \dot{\mathbf{q}})$ the external forces, and $\mathbf{R}$ the contact forces. Vector $\mathbf{q}$ represents the vector of generalized degrees of freedom while $\dot{\mathbf{q}}$ and $\ddot{\mathbf{q}}$ denote the generalized velocity and acceleration vectors respectively.

By using Eq. (1) and a $\theta$ time integrator scheme [21], it is possible to reformulate Eq. (3) in the contact frame. Thus, over a given time interval $\left[t_{i}, t_{i+1}\right]$, the problem is written as a set of transfer equations and interaction laws: (a)

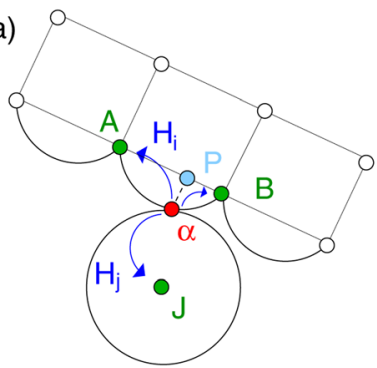

(b)

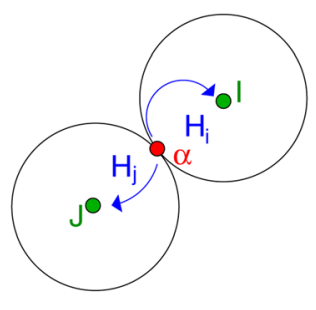

Fig. 1 Representation of the linear mappings between the contact frame (local level) and body frame (global level) for (a) a rigid/deformable contact and (b) a rigid/rigid contact. The red dots represent contact points connected to the local frame while the green dots represent mesh nodes and the center of the mass of particles connected to contact points via the linear mapping $\mathbb{H}$.

$$
\left\{\begin{array}{l}
\mathbb{W} h \mathbf{r}_{i+1}-\mathbf{u}_{i+1}=-\mathbf{u}_{\text {free }} \\
\text { Contact law }\left[\mathbf{r}_{i+1}, \mathbf{u}_{i+1}\right]
\end{array}\right.
$$

where $\mathbb{W}\left(=\mathbb{H}^{*} \tilde{\mathbb{M}}^{-1} \mathbb{H}\right)$ is the Delassus operator that models the local behavior of the solids at the contact points. Equations (4) are written for rigid bodies as well as deformable bodies. If rigid bodies are considered, the couple $\left(\mathbb{M}, \mathbf{u}_{\text {free }}\right)$ is given by:

$$
\left\{\begin{array}{l}
\tilde{\mathbb{M}}=\mathbb{M} \\
\mathbf{u}_{\text {free }}=\tilde{\mathbb{M}}^{-1}\left(h(1-\theta) \mathbf{F}_{i}^{\text {ext }}+h \theta \mathbf{F}_{i+1}^{\text {ext }}\right)
\end{array}\right.
$$

while for deformable bodies, we obtain:

$$
\left\{\begin{array}{l}
\tilde{\mathbb{M}}=\mathbb{M}+h \theta \mathbb{C}+h^{2} \theta^{2} \mathbb{K} \\
\mathbf{u}_{\text {free }}=\tilde{\mathbb{M}}^{-1}\left(h(1-\theta) \mathbf{F}_{i}^{e x t}+h \theta \mathbf{F}_{i+1}^{e x t}\left(\mathbf{q}_{i}+\theta \dot{\mathbf{q}}_{i}, \dot{\mathbf{q}}_{i+1}\right)\right)
\end{array}\right.
$$

In Eq. (5) and Eq. (6), h denotes the time step, $\mathbb{C}$ and $\mathbb{K}$ represent viscosity and stiffness matrices respectively, and $\mathbf{F}^{\text {int }}$ represents the internal forces of the deformable bodies.

2.3 Interaction Laws. To close Eq. (4), it is necessary to define the relation between $\mathbf{u}$ and $\mathbf{r}$ given by the interaction law. This can represent a unilateral contact, an elastic contact, a bilateral relation, or a more sophisticated relation [18].

The interaction should be as realistic as possible in a tribological context. Accounting for cohesion appears essential in order to reproduce physicochemical effects (chemical transformation, oxidation). In dense flows, several authors [24] have shown that plasticity law can be sufficient for understanding rheology, but the role of particle elasticity is not clearly understood. To overcome this problem, two interaction laws are used: a cohesive unilateral contact law and a cohesive elastic contact law (cf. Fig. 2).

With the first law, called IQS (cf. Fig. 2(a)), there is no direct relation between the normal component of the contact force, $r_{N}$, and the gap, $g$, between particles. $\gamma$ denotes the cohesive force necessary to separate two particles acting on the distance $d_{w}$, while the IQS law is described by the linear complementarity problem:

$$
r_{N}(t)+\gamma \geq 0 ; \quad g(t) \geq 0 ; \quad\left[r_{N}(t)+\gamma\right] g(t)=0
$$

For the second law, called ELAS (cf. Fig. 2(b)), a contact stiffness $\mathrm{k}$ is introduced to link $r_{N}$ and $\mathrm{g}$.

$$
r_{N}(t)=-k g(t)+\gamma
$$

In both cases, no local tangential components are considered. However, it is possible to measure a global tangential force related to the cohesive parameter, resulting from particle polydispersity and surface roughness.
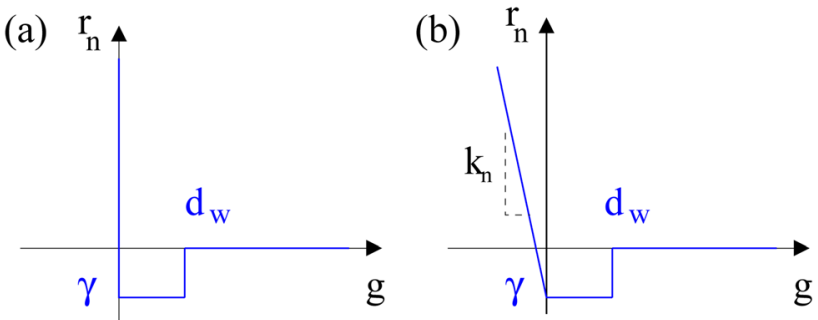

Fig. 2 Plastic law (a), elastic law (b): g represents the interstice between two bodies, $r_{N}$ is the normal reaction of the particle at contact point, $\gamma$ is the cohesive force between two particles in the contact, $d_{w}$ the distance of influence of the cohesion 


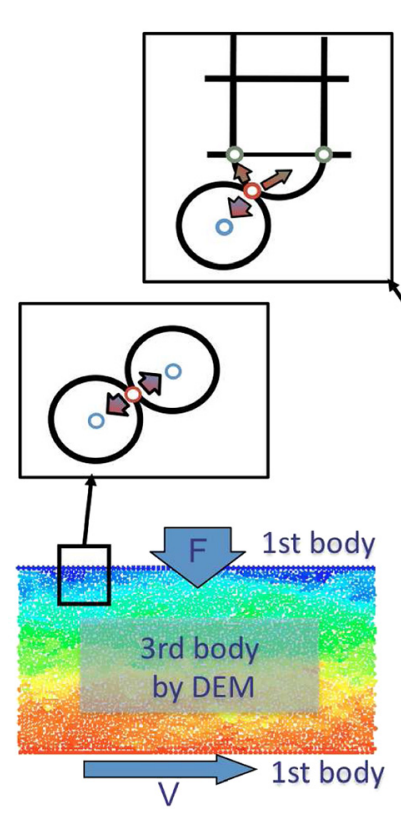

a)

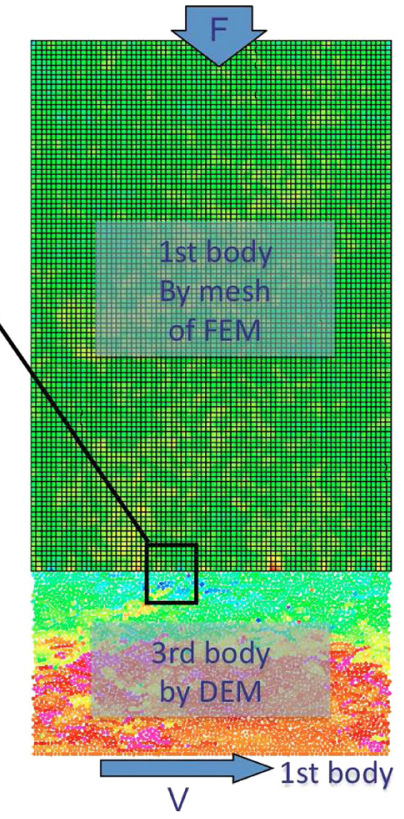

b)
Fig. 3 Geometry of the numerical models used with: (a) a rigid upper body (rigid model), (b) a deformable upper body (deformable model). Visualization of the velocity field within the third body, ranging from 0 (blue color) to $5 \mathrm{~m} / \mathrm{s}$ (red color), the shear velocity value.

\section{Tribological Interface}

3.1 Model Description. The discontinuous and continuous models are coupled in order to understand the evolution of third body flows and the influence of interface modeling. This approach is compared with the classical approach that uses only rigid boundary conditions [25]. In both cases, the model is composed of three parts: an upper and a lower body (UB and LB) and the third body (TB).

In both cases, the third body is composed of 3820 rigid particles with a diameter of $1.2 \times 10^{-6} \mathrm{~m}$ and a variation of $20 \%$. Its thickness $H_{T B}$ is $5 \times 10^{-5} \mathrm{~m}$ while its length $\mathrm{L}$ is $10^{-4} \mathrm{~m}$. The LB is modeled by a collection of 72 rigid particles whose diameter and percent variation are the same as those of the third body.

In the classical simulation (cf. Fig. 3(a)) (rigid upper body), the UB has the same properties as the LB. For the hybrid simulation (cf. Fig. 3(b)) (deformable upper body), the UB is modeled by a deformable structure composed of 7704 quadri- lateral elements. Its thickness $H_{U B}$ is $1.5 \times 10^{-4} \mathrm{~m}$ and its length is $\mathrm{L}$ (i.e $10^{-4} \mathrm{~m}$ ). Its Young modulus, E, is $100 \mathrm{GPa}$ and its Poisson ratio, $\nu$, is 0.33 .
Cell size, $l$, can be chosen (cf. Fig. 4) as a function of smaller diameter of the third body particle, $d$. If $d$ is larger than $l$ (cf. Fig. 4(a)), more than two nodes of the mesh (white dots) are connected to a rigid half disk leading to a loss of mesh accuracy at the interface. If $d$ is smaller than $l$ (cf. Fig. 4(c)), more than two rigid half disks are connected to a single cell (gray dots) and the contact forces are averaged before being transferred to the cell. Finally, in the case where $d=l$ a perfect hybrid element is obtained where a rigid half disk corresponds to a single cell. This latter choice is considered in the simulation with $l$ equal to $10^{-6} \mathrm{~m}$ ) (Table 1).

The densities of the LB, UB, and TB are equal to $7800 \mathrm{~kg} \mathrm{~m}^{-3}$ and gravity is neglected. The boundary conditions are composed of pressure $\mathrm{P}$ of $100 \mathrm{MPa}$, a shear velocity $\mathrm{V}$ equal to $5 \mathrm{~m} \mathrm{~s}^{-1}$ and periodic condition in the direction of the flow applied on the mesh and on the particle to be simulated in an infinite plane.

In the hybrid model, the transfer of information between the $\mathrm{TB}$ and the deformation structure is performed by hybrid contact elements connected to the mesh. With this type of element, roughness is reproduced and the TB can transfer tangential forces (in the sliding direction) to the UB.

3.2 Simulation Results. In the following, the results of twodimensional discrete particle simulations where shear velocity $\mathrm{V}$ is imposed (with periodic boundary used along the shear direction) are presented. Once the contact laws between the particles are fixed, the simulation results depend on different macroscopic parameters: velocity, $\mathrm{V}$, normal pressure, $\mathrm{P}$, density, $\rho$, mean particle diameter, $d$, and cohesion, $\gamma$. The algorithm used in the present study allows reaching higher pressures than those usually simulated $[25,26]$ (due to the iterative process using the nonlinear Gauss Seidel algorithm). Consequently, the results should be compared carefully with the literature on third body simulations usually performed for lower pressures.

3.2.1 Energy Balance. To ensure the validity of the model in terms of energy, it is necessary to check energy conservation during the simulation process. In the model considered, the energy conservation law can be written as:

$$
\frac{d E_{\text {total }}}{d t}=\left(P_{U B}+P_{L B}\right)+P_{T B}
$$

where $P_{U B}$ and $P_{L B}$ represent the power provided by the upper body and the lower body (external power) and $P_{T B}$ that of the third body (internal power), while $d E_{\text {total }} / d t$ represents the time derivative of the kinetic energy. The power of the third body is equal to:

$$
P_{T B}=\sum_{i \in T B} \mathbf{V}_{i} \cdot \mathbf{F}_{i}
$$

where $\mathbf{V}_{i}$ and $\mathbf{F}_{i}$ are respectively the velocity and the force resulting from particle $i$, an element of the third body. The same (a)

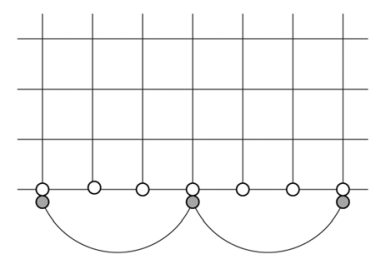

$\mathrm{d}>1$ (b)

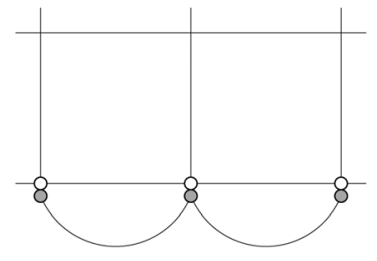

$\mathrm{d}=1$ (c)

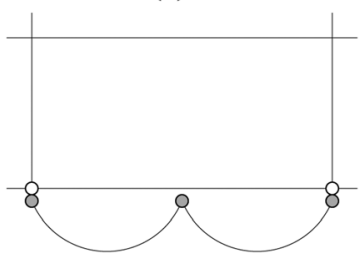

$\mathrm{d}<1$

Fig. 4 Hybrid element as a function of the smaller diameter of a third body particle, $d$, and the size of a cell, $I:(a) d>I,(b) d=I$, and (c) $d<I$ 
Table 1 Simulation parameters

\begin{tabular}{lccccc}
\hline \hline Third body thickness & $H_{T B}$ & $5 \times 10^{-5} \mathrm{~m}$ & Young modulus & $\mathrm{E}$ & $100 \mathrm{GPa}$ \\
Third body length & $\mathrm{L}$ & $10^{-4} \mathrm{~m}$ & Poisson ratio & $\nu$ & 0.33 \\
Particle mean diameter & $\mathrm{d}$ & $1.2 \times 10^{-6} \mathrm{~m}$ & Density & $\rho$ & $7800 \mathrm{~kg} \cdot \mathrm{m}^{-3}$ \\
Shear velocity & $\mathrm{V}$ & $5 \mathrm{~m} \mathrm{~s}^{-1}$ & Upper body & $H_{U B}$ & $1.5 \times 10^{-4} \mathrm{~m}$ \\
Pressure & & $\mathrm{P}$ & $100 \mathrm{MPa}$ & Thickness & \\
\end{tabular}

computation can be performed to obtain the power of the upper body and that of the lower body.

The total kinetic energy $E_{\text {total }}$ of the system is equal to:

$$
\begin{aligned}
E_{\text {total }} & =E_{U B}+E_{L B}+E_{T B} \\
& =\frac{1}{2} m_{U B} V_{U B}^{2}+\frac{1}{2} m_{L B} V^{2}+\sum_{p=1}^{n_{b}} \frac{1}{2}\left(m_{p} V_{p}^{2}\right)
\end{aligned}
$$

where $E_{U B}, E_{L B}$, and $E_{T B}$ are the kinetic energy of the upper, lower, and third bodies respectively. $m_{*}$ and $V_{*}$ denote the mass and the velocity associated with body $*$ and $n_{b}$ is the number of third body particles.

In the simulation following a transient regime, the system reaches steady state when the total kinetic energy of the system is constant. Consequently, its time derivative is equal to zero and the left part of Eq. (10) vanishes.

Moreover, the upper body oscillates around a constant position. Thus its power $P_{U B}$ is null and the Eq. (10) gives the relation $P_{L B}$ $\simeq P_{T B}$. This result is also obtained during the simulation, as presented in Fig. 5. Theoretical and numerical results are in good agreement and underline that all the power supplied by the lower body is completely dissipated in the third body, as shown previously by different authors $[26,27]$.

3.2.2 Global Quantities. Experimentally, the friction coefficient is measured as the ratio between the force in the sliding direction over the force applied on the system. To keep the same kind of measurement in the simulations, the global friction coefficient $\mu(t)$ is defined as:

$$
\mu(t)=\frac{\sum_{i=1}^{n_{L B}} r_{x}^{i}(t)}{F}
$$

where $r_{x}$ is the component of the force in the sliding direction, exerted on one of $n_{L B}$ particles composing the lower boundary, while $\mathrm{F}$ denotes the normal force applied on the upper body.

Figure 6 displays a compilation of simulations performed with different local cohesions, upper body behaviors, and interaction laws. It can be seen that the global friction coefficient depends slightly on the rigidity of the upper body and relies more on the interaction laws. For a given law, the variation of $\mu$ between the rigid and the deformable model is less than $1 \%$. The variation with the interaction law is greater, especially for a high value of $\gamma$, where the variation can reach $16 \%$.

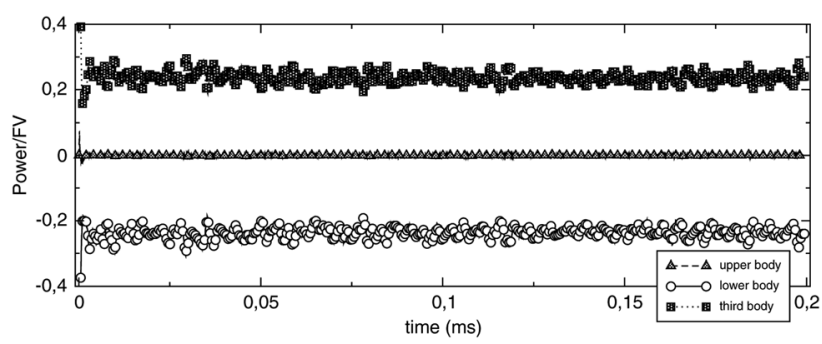

Fig. 5 The power for each bodies in the tribological contact

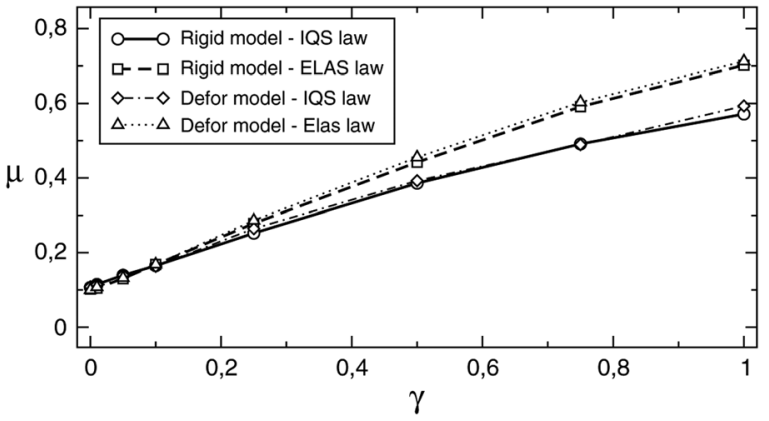

Fig. 6 Evolution of the global friction coefficient $(\mu)$ as a function of the cohesive force $\gamma$ for different upper body behaviors and interaction laws

The results shown in Fig. 6 are unusual. The ELAS law appears more dissipative than the IQS law. To understand such behavior, the quality of the numerical solution is controlled. To perform this control, the error denoted $\varepsilon$, corresponding to the mean distance to the final solution [21] is computed during the simulation:

$$
\varepsilon=\sum_{N \alpha}\left(g_{\alpha}^{c}-g_{\alpha}^{p}\right) / N_{\alpha}
$$

where $N_{\alpha}$ is the number of contacts, $g_{\alpha}^{p}$, the gap between particles computed without contact forces and $g_{\alpha}{ }^{c}$ the gap between particles computed with contact forces. The difference $g_{\alpha}{ }^{c}-g_{\alpha}{ }^{p}$ is equivalent to a residue associated with the resolution of the contact force $\alpha$. With the IQS law, $\varepsilon$ is always lower than $0.1 \%$ of the mean radius, providing good quality for the simulation. This is not the case with the ELAS law. Consequently, the ratio between $\varepsilon_{E L A S}$ and $\varepsilon_{I Q S}$ was plotted for both rigid and deformable models (cf. Fig. 7).

It can be seen that violation for the ELAS law increases with cohesion, especially for value of $\gamma$ greater than 0.1 . To understand this behavior, a basic test was performed with two particles which collide. The energy loss during the shock for different impact velocities was measured (cf. Fig. 8). The size of the particle is equal to that of the sample and the cohesive force was equal to $1 N$.

The theoretical time step $d t^{*}$ is equal to $\sqrt{m / k}$ where $m$ is the effective mass and $k$ the contact stiffness. This time step is usually divided by an arbitrary value $\mathrm{N}$ which corresponds to the discretization of the collision time (usually ranging between 20 and 100 ). For a given velocity, time step $d t$ of the simulation should be shorter than usual in order to preserve energy during the cohesive collision. For example, with an impact velocity of $2 \mathrm{~m} \cdot \mathrm{s}^{-1}$, the time step should be 60,000 times shorter than the theoretical time step $d t^{*}$ to lose less than $0.1 \%$ of the energy. This means that in the simulation campaign performed with the same time

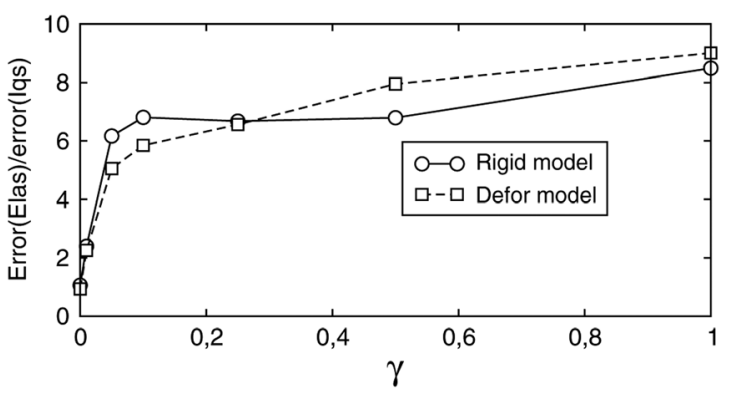

Fig. 7 Evolution of the ratio between $\varepsilon_{E L A S}$ and $\varepsilon_{I O S}$ for both rigid and deformable UB models as a function of the cohesion value 


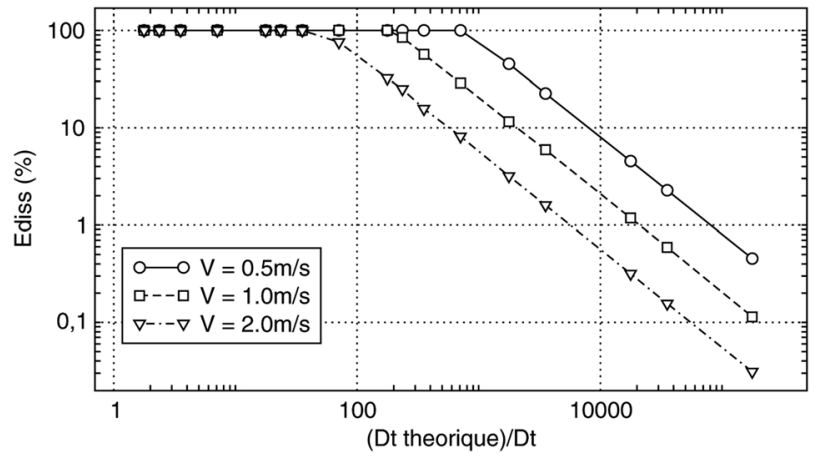

Fig. 8 Percent of energy loss during a shock as a function of the ratio between the theoretical time step and the simulation time step

step for the different cohesion values, global friction is disturbed by the numerical dissipation generated. Consequently, each simulation must be performed with a very short time step to ensure the quality of the results. Nevertheless, this is not done in the following and the results will be restricted to the IQS law, for which result quality can be ensured.

3.2.3 Local Results. To complete the analysis of the global friction coefficient, local analysis is performed across the thickness of the sample corresponding to the gap between boundaries where stress and velocity profiles are measured. The sample is decomposed into several layers with a thickness equal to one particle diameter. For each layer, the mean stress and velocity are measured and time averaged in steady state regime on 500 independent time points. The value of quantity $\mathrm{X}$ (stress or velocity) is obtained by the following formula:

$$
X_{\text {"ayer }_{i}}=\frac{1}{500} \sum_{t=1}^{500} \frac{1}{n L_{i}\left(t^{k}\right)} \sum_{j \in \text { laye }_{i}} X_{j}\left(t^{k}\right)
$$

where $n_{L i}$ is the number of particles in the layer $i$ at time $t^{k}$.

The stress tensor of a particle $\mathrm{P}$, denoted $\sigma(P)$, is defined as is typical for granular material [28]:

$$
\sigma_{i j}(P)=\frac{1}{\operatorname{vol}(P)} \sum_{\alpha \in \mathcal{L}_{p}} r_{i}^{\alpha} l_{j}^{\alpha}
$$

where $r^{\alpha}$ is the force exerted on contact $\alpha, l$ the distance between the contact point and the center of mass of particle $P$ and $L_{p}$ the contact list of particle $P$.
To see the influence of the rigidity of the upper body on third body flows, three types of upper body were used: a rigid model and two deformable models with different Young moduli of 100 $G p a\left(E_{1}\right)$ and $3 G p a\left(E_{2}\right)$ respectively.

When the profile of the $\sigma_{y y}$ component is observed (cf. Fig. 9), no difference can be seen between the rigid and deformable models for the UB. The $\sigma_{y y}$ component is always constant through the thickness (equal to $100 \mathrm{Mpa}$ the given pressure) for $\gamma$ equal to 0 (cf. Fig. 9(a)), 0.5 (cf. Fig. 9(b)), and 1 (cf. Fig. 9(c)).

When the profile of the $\sigma_{x y}$ component is observed (cf. Fig. 10), no difference can be observed for the different models for the UB. Variation occurs when $\gamma$ changes. For $\gamma$ equal to $0,0.5$ and $1 N, \sigma_{x y}$ is equal to 5,32 , and $50 M P a$ respectively (with the IQS law).

Consequently, as with the global friction coefficient, first body rigidity does not influence the stress profile through the thickness of the third body. Such behavior may be related to the fact that the stress profiles and global friction coefficient are directly linked to normal and tangential forces within the system. When averaging the whole system, no global variation occurs in the case where first body rigidity influences force values locally; thus mechanical equilibrium is conserved.

To complete the analysis, the velocity profiles across the thickness are observed. Figure 11 presents the visualization of the sample in different configurations while Fig. 12 presents the velocity profiles across the thickness in steady state.

Two different colors (blue and maroon) are given to the particles in order to emphasize the shear rate in the third body. In the initial state of the structure, the third body of the two models is divided into two regular areas (blue and maroon). When the local cohesion force $\gamma$ is null, the velocity profiles are quasi-linear (fluid regime). When $\gamma$ increases, the velocity profiles are sensitive to the rigidity of the upper body. Asymmetries appear in the velocity profile, and according to the rigidity, the profile can be radically different, increasing as $\gamma$ increases. For high UB rigidity values, the accommodation of velocity is localized at the TB/UB interface as well as at the TB/LB interface. When the rigidity of the upper body decreases, accommodation of the deformation at the UB/TB interface can be observed, especially for high cohesion values, leading to velocity accommodation at the TB/LB interface. The main consequence of this phenomenon is the localization of dissipation within the sample. When performing thermo-mechanical coupling $[29,30]$, changes of localization in the model lead to changes of temperature profile and the possibility of poor heat distribution in the sample.

Note that Fig. 12(c)) could be confusing. One can observethat the $\mathrm{TB} / \mathrm{UB}$ velocity accommodation is less for the absolutely rigid case than the high UB rigidity value case (E1). This effect is probably due to the fact that, for the high UB rigidity value case, (a)

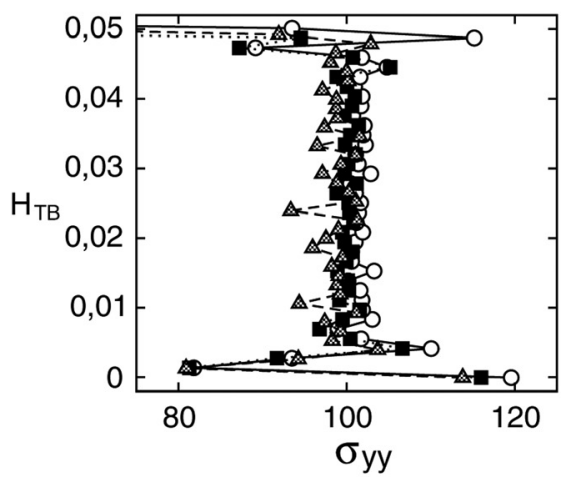

(b)

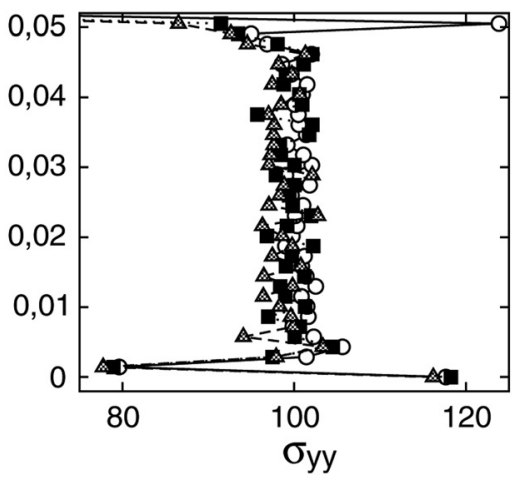

(c)

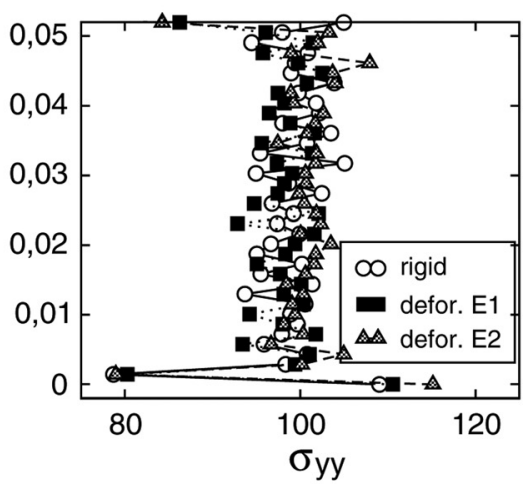

Fig. 9 Profile of $\sigma_{y y}$ for the IQS law through the third body thickness as a function of upper body rigidity and the local cohesive force $\gamma:(a) \gamma=0,(b) \gamma=0.5$, and $(c) \gamma=1$ 
(a)

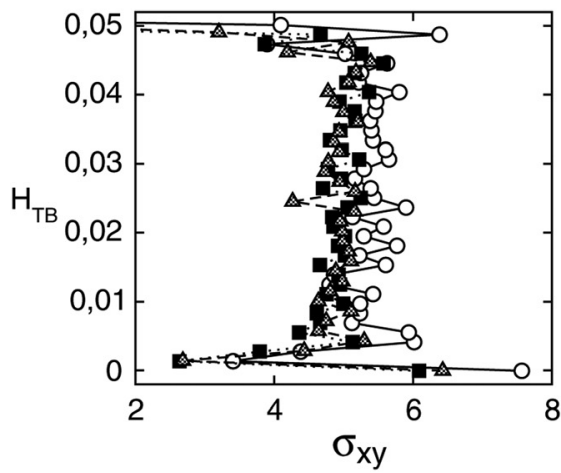

(b)

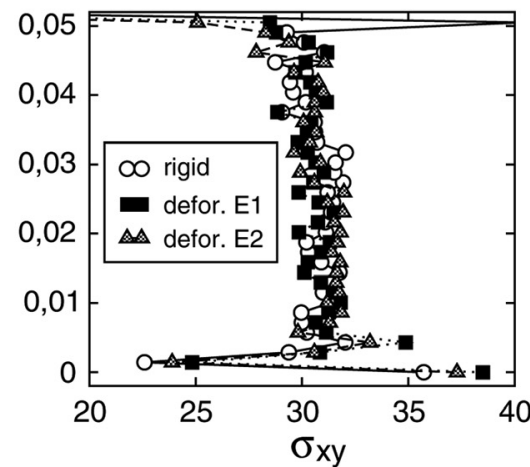

(c)

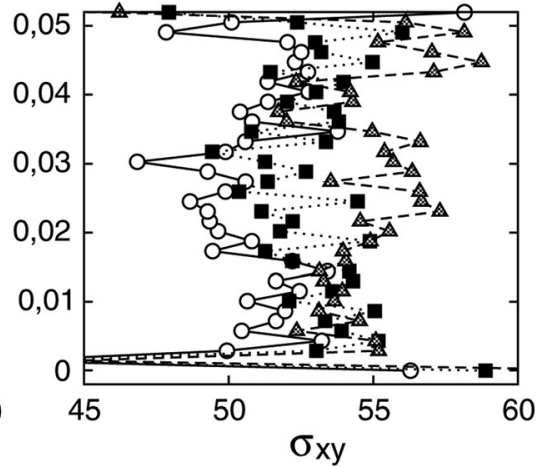

Fig. 10 Profile of $\sigma_{x y}$ for the IQS law through the third body thickness as a function of the upper body rigidity and the local cohesive force $\gamma:(a) \gamma=0,(b) \gamma=0.5$, and $(c) \gamma=1$
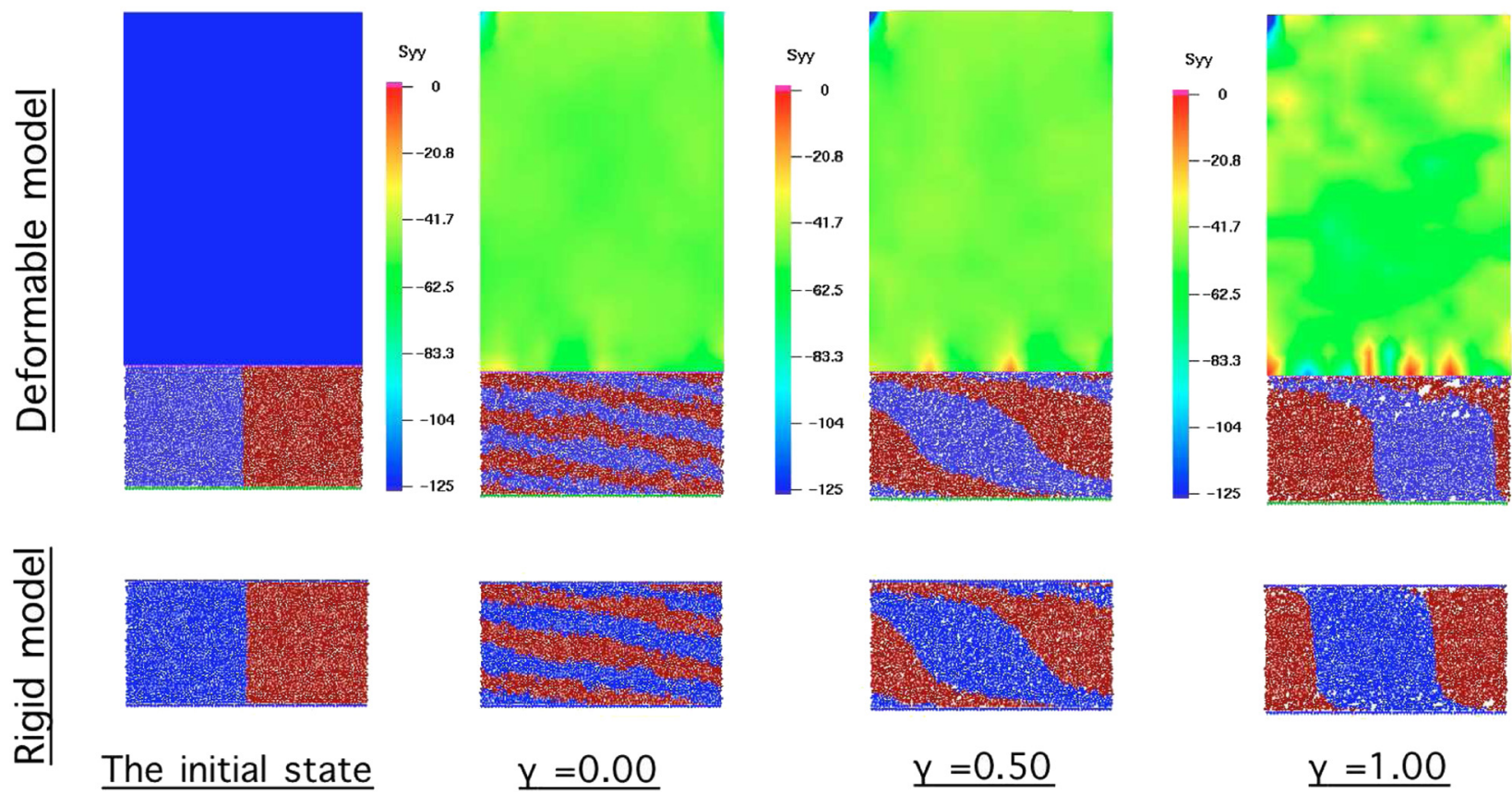

$$
\underline{y}=0.00
$$

$$
\underline{Y}=0.50
$$

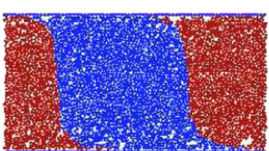

$y=1.00$

Fig. 11 Visualization of the final snapshot of the simulation for different cohesive forces: $\sigma_{y y}$ component for the deformable structure and deformation pattern of the third-body

(a)

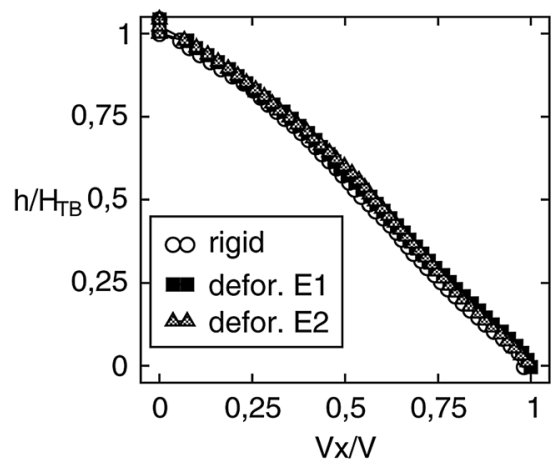

(b)

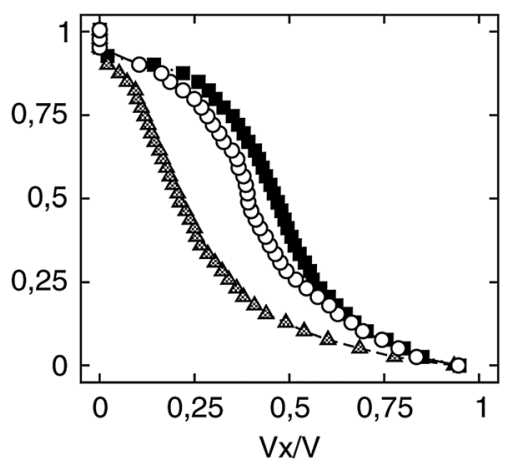

(c)

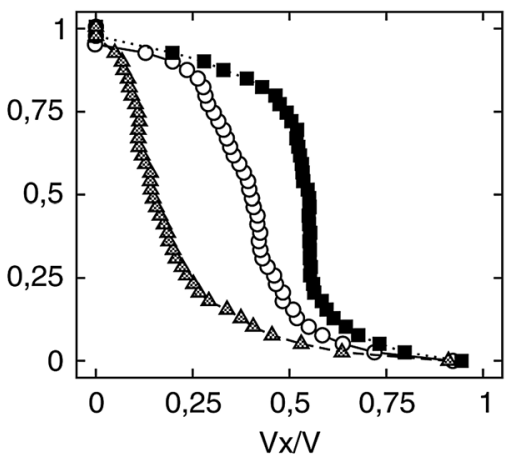

Fig. 12 Velocity profile through the third body thickness as a function of the upper body rigidity and the local cohesive force $\gamma$ 
the velocity accommodation occurs alternately at the TB/UB interface and at the TB/LB interface. This phenomenon leads to asymmetric velocity profile. More investigations could be performed to fully understand this trend.

\section{Conclusions and Outlook}

The present paper proposes a hybrid approach, combining both continuous description related to finite element modeling, and discontinuous description related to rigid discrete element modeling, to underline the influence of first body rigidity on third body rheology. From the numerical viewpoint, the simulation underlined the fact that the interaction law has a strong effect on the third body rheology. Moreover, the time step of the simulations must be chosen carefully when cohesion laws are used. A heuristic approach was proposed to determine the time step value. From a physical viewpoint, the simulation results demonstrated that the velocity profile could be different, even if the global friction coefficient was the same for two different bulk behaviors of the first body (rigid or deformable). This difference can have a strong influence on the localization of energy dissipation and therefore influence the temperature profile [30].

\section{Nomenclature}

$E_{c}=$ kinetic energy of the body

$E_{\text {total }}=$ kinetic energy total of the system

$P_{U B}=$ power due to the upper body

$P_{L B}=$ power due to the lower body

$P_{T B}=$ power due to the third body

$F=$ normal force applied on the upper body

$V=$ shear velocity applied on the lower body

$L=$ dimension of contact interface in $\mathrm{X}$ direction

$H_{T B}=$ thickness of the third body

$H_{U B}=$ thickness of the upper body

$E=$ young modulus of the upper body

$\nu=$ poisson ratio of the upper body

$m_{i}=$ mass of the particle $\mathrm{i}$

$d=$ mean particle diameter

$d t=$ time step

$g=$ gap between particles

$\gamma=$ local cohesion force

$k=$ local contact stiffness

$\mathbf{r}=$ local contact force

$\mathbf{u}=$ local relative velocity

$\mathbf{R}=$ global contact force

$\mathbf{q}=$ configuration parameter

$\dot{\mathbf{q}}=$ global velocity

$\mathbb{M}=$ mass matrix

$\mathbf{F}^{\text {int }}=$ internal force and the nonlinear inertia terms

$\mathbf{F}^{\text {ext }}=$ external forces

$\mathbb{W}=$ delassus operator

$\mathbb{H}=$ linear mapping

$\mathbf{u}_{\text {free }}=$ local relative velocity without contact forces

$\mu=$ global friction coefficient

$\mu_{\text {mean }}=$ mean global friction coefficient

$\sigma_{x y}=$ stress field in xy direction

$\sigma_{y y}=$ stress field in yy direction

\section{Acknowledgment}

Part of this work was funded by the Agence Nationale de la Recherche in the framework of the project ANR-08-JCJC0020-01.

\section{References}

[1] Godet, M., 1984, "The Third-Body Approach: A Mechanical View of Wear," Wear, 100, pp. 437-452.

[2] Berthier, Y., 1990, "Experimental Evidence for Friction and Wear Modelling," Wear, 139, pp. 77-92.

[3] Weinzapfel, N., and Sadeghi, F., 2009, "A Discrete Element Approach for Modeling Cage Flexibility in Ball Bearing Dynamics Simulations," ASME J. Tribol., 131, p. 021102.

[4] Massi, F., Giannini, G., and Baillet, L., 2006, "Brake Squeal as Dynamic Instability: An Experimental Investigation", J. Acoust. Soc. Am., 120(3), pp. $1388-1399$

[5] Berthier, Y., Vincent, L., and Godet, M., 1988, "Velocity Accommodation in Fretting," Wear, 125(1-2), pp. 25-38.

[6] Berthier, Y., 2005, Wear - Materials, Mechanisms and Practice, John Wiley \& Sons, Ltd., Chap. 12.

[7] Sawyer, W., and Tichy, J., 2001, "Lubrication With Granular Flow: Continuum Theory, Particle Simulations, Comparison With Experiment," ASME J. Tribol., 123, pp. 777-784.

[8] Higgs III, C. F., and Tichy, J., 2004, "Granular Flow Lubrication: Continuum Modeling of Shear Behaviour," ASME J. Tribol., 126, pp. 499-509.

[9] Yu, C.-M., Craig, K., and Tichy, J., 1994, "Granular Collision Lubrication," J. Rheol., 38(4), pp. 921-936.

[10] Hou, K., Kalousek, J., and Magel, E., 1997, "Rheological Model of Solid Layer in Rolling Contact," Wear, 211, pp. 134-140.

[11] Zhou, L., and Khonsari, M., 2000, "Flow Characteristics of a Powder Lubricant Sheared Between Parallel Plates," ASME J. Tribol., 122(1), pp. 147-155.

[12] Elrod, H., and Brewe, D., 1991, "Numerical Experiments With Flows of Elongated Granules," Tribol. S., 21, pp. 219-226.

[13] Lubrecht, A., and Berthier, Y., 1995, "Granular Lubrication; a Simple Model and Trends," Tribol. S., 30, pp. 53-62.

[14] Ghaouti, A., Chaze, M., Dubujet, P., and Sidoroff, F., 1996, "Particulate and Granular Simulation of the Third Body Behaviour," Tribol. S., 31, pp. $355-365$.

[15] Seve, B., Iordanoff, I., and Berthier, Y., 2001, "A Discrete Solid Third Body Model: Influence of the Inter Granular Forces on the Macroscopic Behaviour," Tribol. S., 39, pp. 361-368.

[16] Iordanoff, I., Berthier, Y., Descartes, S., and Heshmat, H., 2002, “A Review of Recent Approaches for Modeling Third Bodies,” ASME J. Tribol., 124, pp. 725-735.

[17] Fillot, N., Iordanoff, I., and Berthier, Y., 2004, "A Granular Dynamic Model for the Degradation of Material,” ASME J. Tribol., 126(3), pp. 606-614.

[18] Renouf, M., Saulot, A., and Berthier, Y., 2006, "Third Body Flow During a Wheel-Rail Interaction," Proc. 3rd European Conference on Computational Mechanics Solids, C. A. M. S. et al, eds., Lisboa, Portugal, CD-ROM.

[19] Kabir, A., Jasti, V., Higgs III, C. F., and Lovell, M., 2008, "An Evaluation of the Explicit Finite-Element Method Approach for Modelling Dense Flows of Discrete Grains in a Couette Shear Cell," I. Mech. E, 222, pp. 715-723.

[20] Moreau, J. J., 1994, "Some Numerical Methods in Multibody Dynamics: Application to Granular Materials," Eur. J. Mech. A Solids, 13(4-suppl.), pp. 93-114.

[21] Jean, M., 1999, "The Non Smooth Contact Dynamics Method," Comput. Meth. Appl. Math. Engrg., 177, pp. 235-257.

[22] Saussine, G., Cholet, C., Dubois, F., Bohatier, C., Gautier, P., and Moreau, J. J., 2006, "Modelling Ballast Behaviour Under Dynamic Loading. Part 1: A 2D Polygonal Discrete Element Method Approach," Comput. Meth. Appl. Mech. Engrg., 195(19-22), pp. 2841-2859.

[23] Acary, V., and Jean, M., 2000, "Numerical Modeling of Three Dimensional Divided Structures by the Non Smooth Contact Dynamics Method: Application to Masonry Structure," Proc. 5th International Conference on Computational Structures Technology, B. Topping, ed., Civil-Comp Press, pp. 211-222.

[24] Chevoir, F., Roux, J.-N., da Cruz, F., Rognon, P., and Koval, Jr., G., 2009, "Friction Law in Dense Granular Flows," Powder Tech., 190(1-2), pp. 264-268.

[25] Iordanoff, I., Sève, B., and Berthier, Y., 2002, "Solid Third Body Analysis Using a Discrete Approach: Influence of Adhesion and Particle Size on the Macroscopic Behavior of the Contact," ASME J. Tribol., 124, pp. 530-538.

[26] Richard, D., Iordanoff, I., Berthier, Y., Renouf, M., and Fillot, N., 2007, "Friction Coefficient as a Macroscopic View of Local Dissipation," ASME J. Tribol., 129(4), p. 031404

[27] Iordanoff, I., Fillot, N., and Berthier, Y., 2005, "Numerical Study of a Thin Layer of Cohesive Particles Under Plane Shearing," Powder Tech., 159(1), pp. 46-54.

[28] Radjai, F., Jean, M., Moreau, J.-J., and Roux, S., 1996, "Force Distributions in Dense Two-Dimensional Granular Systems," Phys. Rev. Lett., 77(2), pp. 264-277.

[29] Richard, D., Iordanoff, I., Renouf, M., and Berthier, Y., 2008, "Thermal Study of the Dry Sliding Contact With Third-Body Presence," ASME J. Tribol., 130(3), p. 031404.

[30] Renouf, M., Cao, H.-P., and Nhu, V.-H., 2011, "Multiphysical Modeling of Third-Body Rheology," Tribol. Inter., 44(4), pp. 417-425. 